\title{
High potency factor VIII concentrates: value not proved?
}

\author{
John D Cash
}

In June 1988 concern was expressed at events in the marketplace designed to promote the use of high potency, high cost factor VIII concentrates.' At that time there was no evidence to support the marketing claims being made-that these concentrates had a clinically relevant sparing effect on the immune system in patients with haemophilia. It was concluded that these therapeutic products, of unproved additional clinical value when compared with lower potency products, would substantially and unnecessarily increase the cost of haemophilia care.

\section{Clinical benefit?}

In 1991 despite intensive international research we must conclude that there is still no substantive scientific evidence to support the central marketing theme for high potency factor VIII concentrates. Neither, surprisingly, has convincing clinical evidence emerged that these products are of special benefit (in terms of influencing the natural progression of disease) to patients who are HIV positive. On the other hand it would seem certain that some of these concentrates can be of real value in a small group of patients who develop minor (but clinically important) "allergic" reactions to some intermediate factor VIII concentrates. They can also have genuine convenience value in providing smoother 24 hour control of factor VIII levels in the major surgical setting. Beyond this, preliminary (as yet unconfirmed) data suggest that some high potency products may be associated, particularly in children, with a higher than expected prevalence of factor VIII inhibitor formation ${ }^{2-1}-\mathbf{a}$ clinical event that can have dire consequences for the patients.

There is little doubt that, in a climate where most doctors caring for haemophiliac patients have been particularly distressed by the consequences of HIV infection in many of the patients, the marketing campaigns exerted by those companies developing high potency factor VIII concentrates have been difficult to avoid. Thus we have seen in many parts of the world the hasty issue of product licences by medicine control authorities, and the marketing exercises have been further stepped up, to the extent that some patients have been directly targeted with advertising. A succession of almost identical parliamentary questions from all parts of Britain would suggest that these campaigns have even reached members of parliament, ${ }^{5-x}$ and the Department of Health has inadvertently added its support by exhortations on clinical freedom of prescribing. ${ }^{9}$ Many of these activities could be ascribed to a desire to turn away from those dark days in the early 1980 s when so many patients contracted HIV, and turn towards a brighter tomorrow.

But now the cost of this putative development must be fully addressed. The extra bill for products currently available in the United Kingdom could be more than $£ 10 \mathrm{~m}$ a year. For some individual haemophiliac patients the annual increase in cost will be about
$£ 10000$. Many of us might not demur at this increased cost if it was of proved clinical benefit, but that benefit, except in a very small group of patients, has hitherto proved to be a mirage. Such observations also need to be set in the context of another mirage - the freedom to prescribe against a background of cash limits on pharmacy budgets. Many patients, denied access to drugs of proved value by these cash limits, are looking for annual incremental costs well below those required to switch haemophiliac patients to the currently licensed high potency factor VIII concentrates; others are prevented from entry into important clinical trials.

\section{A code of practice}

Most manufacturers of pharmaceuticals have an instinctive, market led urge to improve their products, particularly with regard to potency, and the directors of haemophilia centres in the United Kingdom have recently supported such innovations. But in the United Kingdom a code of practice for this type of exercise has been established by the Association of the British Pharmaceutical Industry. The most relevant sections of this code, in the context of the factor VIII concentrate debate, are:

- Claims for superior potency per weight are meaningless and are best avoided unless they can be linked with some practical advantage - for example, reduction in side effects or cost of effective dosage

- Claims should not imply that a medical product or active ingredient has some special merit, quality, or property unless this can be substantiated.

It is difficult not to conclude that at the present time the introduction of the currently available high potency factor VIII concentrates does not meet with the basic tenets of this code.

Many will advise that, in view of the current overriding imperative for the development in the NHS of market forces responding to customer demands, we should plan now for the imminent widespread use of high potency factor VIII concentrates. Others, however, who are particularly concerned with the consequences of cash limits on pharmacy budgets, would advise that any movement in this direction should be heavily influenced by cost-benefit considerations.

Cost-benefit considerations could prove to be less urgent if the plasma fractionators were able to develop technical approaches that delivered high potency factor VIII concentrates at manufacturing costs comparable with intermediate potency concentrates. Recent evidence suggests that this may be possible. ${ }^{10}$ Further, this technology does not involve immunopurification and thus the resultant product will not be contaminated with trace quantities of mouse proteins. This development must surely be good news for haemophiliac patients, their hard pressed prescribing physicians, and anxious health service managers who 
are currently so preoccupied with the funding implications of change. But rejoicing may yet be premature, for the spectre of a higher prevalence of factor VIII inhibitors in patients receiving high potency concentrates needs to be looked at and, we hope, dispelled.

1 Cash JD. Coagulation factor VIII concentrates and the marketplace. Lancet 1988:i: 1270 .

2 Bell BA, Kurczynski EM, Bergman G. Inhibitors to monoclonal antibody purified factor VIII. Lancet 1990;ii:638.

3 Kessler GM, Sachse K. Factor VIII: C inhibitor associated with monoclonalantibody purified factor VIII concentrate. Lancet 1990;i:1403.

4 Montoro JB, Rodriguez S, Altisent C, Tusell JM. Transient factor VIII inhibitor and treatment with monoclonal-antibody-purified factor VIUL Lancet 1991;ii: 1222

5 House of Commons official report (Hansard): 199] January 24;184:col 307. (No 41.)

6 House of Commons official report (Hansard) 1991 February 12;185:col 401. (No 55 .)

House of Commons official report (Hansard) 1991 February 14;185:col 572. (No 57.)

8 House of Commons official report (Hansard) 1991 February 19;186:col 86. (No 59.)

9 Cash JD. Supply of blood products. BMF 1991;302:849.

10 Burnouf T, Burnouf-Radosevich M, Huart JJ, Goudemand M. A highly purified factor VIII: c concentrate prepared from cryoprecipitate by ion exchange chromatography. Vox Sang 1991;60:8-15.

(Accepted 30 fuly 1991)

\section{For Debate}

\section{Guidelines for the management of convulsions with fever}

Joint Working Group of the Research Unit of the Royal College of Physicians and the British Paediatric Association

A workshop on the subject of childhood convulsions with fever was held at the Royal College of Physicians, London, on 3 July 1990 under the joint auspices of the Research Unit of the Royal College of Physicians and the British Paediatric Association. There were 21 invited participants, of whom 11 prepared background papers on various aspects of the subject (appendix). The background papers were circulated before the meeting, and after discussion, which was often brisk, this report was agreed.

\section{Definition}

The working group considered that it was important to distinguish "convulsions with fever" and "febrile convulsions." Convulsions with fever include any convulsion in a child of any age with fever of any cause. For the purposes of clinical management the term febrile convulsions should be limited to "an epileptic seizure occurring in a child aged from six months to five years, precipitated by fever arising from infection outside the nervous system in a child who is otherwise neurologically normal." The working group considered that it was proper to use the term "epileptic" in so far as the neurophysiological substrate of a febrile convulsion is a paroxysmal neuronal discharge, as in an epileptic seizure.

Among children who have convulsions with fever are those with pyogenic or viral meningitis, herpes simplex encephalitis, other acute encephalitides, cerebral palsy with intercurrent infection, and metabolic or neurodegenerative disease with a seizure precipitated by fever. Children who have a prolonged seizure or who have not completely recovered within one hour should be suspected of having one of these conditions and investigated accordingly.

Research Unit of Royal College of Physicians and British Paediatric Association, London Members of the joint working group are listed in the appendix

Correspondence to: Dr A Hopkins, Director, Research Unit, Royal College of Physicians, Regent's Park, London NW1 4LE.

\section{Primary care}

Much discussion centred on the question of when children with fever and convulsions should be admitted to hospital. Some members of the working group thought that all should be admitted after a first convulsion, mainly because of anxiety about overlooking meningitis. Most members thought that some selection was possible. The following factors would favour admission after a first convulsion: $(a)$ a complex convulsion - that is, one lasting longer than 20 minutes, with focal features, repeated in the same episode of illness or with incomplete recovery after one hour; $(b)$ a child aged less than 18 months; $(c)$ early review by a doctor at home not possible; $(d)$ home circumstances inadequate, or more than usual parental anxiety, or parents' inability to cope. It should be remembered that a history of previous convulsions does not rule out the possibility of meningitis.

\section{Investigations}

No investigations are routinely necessary in all children after a febrile convulsion. It is prudent to measure the blood glucose concentration with a glucose oxidase strip in any child who is still convulsing or unrousable when seen either with or without fever. Other investigations will be determined by the febrile illness rather than by the convulsion, with the possible exception of lumbar puncture.

\section{LUMBAR PUNCTURE}

The possibility of failing to diagnose meningitis worries most doctors. The working group did not consider that all children who convulse with fever should have a lumbar puncture. A lumbar puncture should, however, be performed if there are clinical signs of meningism; after a complex convulsion; if the child is unduly drowsy or irritable or systemically ill; or if the child is aged less than 18 months (probably) and almost certainly if the child is aged less than 12 months. Ideally the decision should be taken by an experienced doctor, who may decide on clinical grounds that lumbar puncture is unnecessary even in a younger child, but when in doubt the investigation should be performed. The doctor deciding not to undertake a lumbar puncture should be prepared to review the decision within a few hours.

A comatose child must be examined by an experienced doctor before lumbar puncture because of the risk of coning.' Brain imaging may be necessary.

\section{ELECTROENCEPHALOGRAPHY}

An electroencephalogram is not a guide to treatment or to prognosis. It is not helpful after single or recurrent febrile convulsions. It may help if the clinical picture suggests pathological changes in the brain, but in this case computed tomography or magnetic resonance imaging will usually be performed first. 